\title{
Model of control over cutting tool demand in a machining shop
}

\author{
Igor Lutoshkin ${ }^{1}$, Aleksandr Madanov, ${ }^{2, *}$, and Yury Polyanskov ${ }^{3}$ \\ ${ }^{1}$ Ulyanovsk State University, Department of Digital Economy, 432017 Ulyanovsk City, L'va Tolstogo \\ st, h.42, Russian Federation \\ ${ }^{2}$ Ulyanovsk State University, Scientific Research Centre of CALS-technologies, 432017 Ulyanovsk \\ City, L'va Tolstogo st, h.42, Russian Federation \\ ${ }^{3}$ Ulyanovsk State University, Department of Mathematical Modeling of Engineering Systems, 432017 \\ Ulyanovsk City, L'va Tolstogo st, h.42, Russian Federation
}

\begin{abstract}
The article proposes an optimization model of control over cutting tool demand in a machining shop. The model is based on application of one of the optimization problems, which occur at an enterprise depending on its current requirements: minimization of time and financial costs, and with convolution of these criteria. Furthermore, the work describes a software program used for calculation of required quantity of cutting tools necessary for meeting of production targets.
\end{abstract}

In an environment of limited demand for manufactured produce and unutilized capacity, the main objective of concerned parties consists in "improvement of performance efficiency of machine-building enterprises through reduction of manufacturing and non-manufacturing costs."

Assessment of performance of a manufacturing facility involves analysis of a big number of various heterogeneous sub-systems that affect overall performance. Principles of effective performance of an enterprise are chosen considering general idea and strategy. Efficient techniques of assessment and management of the enterprise performance are particularly important for Russian aircraft manufactures in the current environment of economic constraints, changing economic model (digital transformation), development of new manufacturing processes and shaping of new requirements for competence of personnel. Integration of the following principles of assessment and management becomes relevant: just-in-time, at specified cost price, allowing for risks.

In their work, enterprises apply statistical and standard calculation methods of rates of use of cutting tools.

For mass and large-scale production, rate of cutting tool use per program is defined with a calculation method [1] and formula (1):

$$
H_{\mathrm{u}}=\frac{N_{\mathrm{p}} t_{c} i}{60 T_{w}\left(1-K_{\mathrm{fr}}\right)}
$$

\footnotetext{
* Corresponding author: madanov.alexandr@yandex.ru
} 
where $H u$ is use of a certain standard-size cutting tool, units; $N p$ is a number of parts machined with this tool by annual program, units; tc is cutting time for this operation, min; $i$ is a number of tools of this standard-size used at the same time; $T w$ is time of a tool work until wear-out, h; Kfr is premature failure rate of a tool (that equals 0.05 ).

For piece- and one-off production, exaggerated rate of tool use is determined [1], for example, for 1000 machine-hours of work for a certain type of equipment:

$$
H_{\mathrm{u}}=\frac{1000 K_{c} K_{\mathrm{ut}} i}{T_{\mathrm{w}}\left(1-K_{f r}\right)}
$$

where $H u$ is use rate of a certain standard-size cutting tool, units; $K c$ is cutting time rate of the total period of tool operation; Kut is usability rate of this standard-size tool in the total machine hours; $i$ is a number of tools of this type used at the same time; $T w$ is time of a tool work until wear-out, h; $K f r$ is premature failure rate of a tool (that equals 0.05 ).

Statistical method applies report data on actual tool wear during a certain period (year or several years). Rate of use of a cutting tool is defined as:

$$
H_{u}=\frac{\left(\mathrm{C}_{f u}-\mathrm{C}_{\mathrm{unj}}\right) K_{\mathrm{a}}}{1000}
$$

where $C f u$ is actual use during a period; $C u n j$ is unjustified non-conformance of use of a tool; $K a$ is an influence rate of activities aimed at reduction of tool use $(K a \leq 1)$.

Using these methods does not lead to full realization of principles - just-in-time and at specified cost price - when planning production.

The paper [2] introduces an automated prediction method for the life of cutting tools for CNC machine tools. The method is based on data of technological processes and information on cutting tool durability. However, this work does not consider specifics of tool assembly and possible application of the same tool item simultaneously on different machines.

Let us consider a model of product cost management through definition of optimum values of factors that condition a choice of cutting tools in the machining shop. Model input parameters include monthly production schedule of a given machining shop and a set of numerical control (NC) programs and CAM-projects for produce manufacturing. A list of cutting tools required for meeting of production targets is an output characteristic.

Let us introduce variables $q_{i}, 1 \leq i \leq n: q_{i}$ is a number of products of $i$ type targeted for production.

For each product type $i$ there is a set of NC programs ensuring manufacturing of these products. Assume $k_{i}$ is a number of programs for manufacturing of a product type $i$. Each program is appointed a set of cutting tools, which is used to generate tool assembly for the equipment defined by this program. It is clear that the cost of tool assembly and its operational lifetime could be defined based on the set of cutting tools.

Write $\tau_{i j}$ for time that it takes to machine one unit of $i$ product with $j$ program. Assume that while machining, a manufacturing process is linear, multistage and sequential in its effect, meaning it could be sequentially applied to several different tool assemblies. Assume $a_{i j s}$ is a number of tool assemblies required for manufacturing of a single unit of $i$ product with $j$ program at stages $s, 1 \leq i \leq n, 1 \leq j \leq \mathrm{k}_{\mathrm{j}}, 1 \leq s \leq m_{i j}$. Assume that amount $a_{i j s}$ is generically expressed in fractional numbers, and $\tau_{i j}$ is evaluated in hours.

Assume a control task consists in estimation of a number of calls of NC programs for meeting the production targets of a shop. Assume $x_{i j}$ is a number of calls of $j$ program for manufacturing of $i$ product. Under the assumption that one NC program ensures manufacturing of one product unit, a balance relation is true: 


$$
x_{i 1}+x_{i 2}+\ldots+x_{i k_{i}}=q_{i}
$$

In addition, $x_{i j} \geq 0,1 \leq i \leq n, 1 \leq j \leq k_{i}$. This balance relation is realized for all types of produce, or for $1 \leq i \leq n$.

Within the introduced notations, a total number of tool assemblies for manufacturing of $i$ product with $j$ program at $s$ stage is expressed through $a_{i j s} x_{i j}$. If we introduce a cost $p_{i j s}$ of one tool assembly for a $i$ product with the $j$ program at $s$ stage, then total costs $C$ of tool assembly generation are expressed through a relation:

$$
C\left(x_{11}, x_{12}, \ldots, x_{1 k_{1}}, \ldots, x_{n 1}, x_{n 2}, \ldots, x_{n k_{n}}\right)=\sum_{i=1}^{n} \sum_{j=1}^{k_{i}} \sum_{s=1}^{m_{i j}} p_{i j s} a_{i j s} x_{i j}
$$

Total time expenditure $T$ could be calculated based on relation:

$$
T\left(x_{11}, x_{12}, \ldots, x_{1 k_{1}}, \ldots, x_{n 1}, x_{n 2}, \ldots, x_{n k_{n}}\right)=\sum_{i=1}^{n} \sum_{j=1}^{k_{i}} \tau_{i j} x_{i j}
$$

Selection of a set of variables $x_{i j}\left(1 \leq i \leq n, 1 \leq j \leq k_{i}\right)$ defines financial and time expenditures. If balance relations are valid, minimizing a function of expenditures $C$ could result in a situation where production target is not met at a reasonable time. Whereas minimizing a function of time expenditure $T$ could significantly increase expenses for cutting tool procurement. In such event, it is reasonable to reduce these criteria to a single one or minimize one of the criterion while the level of another is fixed.

For instance, we could fix an acceptable level of expense $C^{*}$ for cutting tool procurement. Then a task

$$
\left\{\begin{array}{l}
T\left(x_{11}, x_{12}, \ldots, x_{1 k_{1}}, \ldots, x_{n 1}, x_{n 2}, \ldots, x_{n k_{n}}\right) \rightarrow \min , \\
C\left(x_{11}, x_{12}, \ldots, x_{1 k_{1}}, \ldots, x_{n 1}, x_{n 2}, \ldots, x_{n k_{n}}\right) \leq C^{*}, \\
\sum_{j=1}^{k_{i}} x_{i j}=q_{i}, \quad 1 \leq i \leq n, \\
x_{i j} \geq 0, \quad 1 \leq i \leq n, \quad 1 \leq j \leq k_{i} ; \quad x_{i j} \in\{0\} \bigcup N ;
\end{array}\right.
$$

ensures minimization of time expenditure at a specified cost price.

If time expenditure is fixed, another task could be minimizing cost price at fixed time level $T^{*}$ :

$$
\left\{\begin{array}{l}
C\left(x_{11}, x_{12}, \ldots, x_{1 k_{1}}, \ldots, x_{n 1}, x_{n 2}, \ldots, x_{n k_{n}}\right) \rightarrow \min \\
T\left(x_{11}, x_{12}, \ldots, x_{1 k_{1}}, \ldots, x_{n 1}, x_{n 2}, \ldots, x_{n k_{n}}\right) \leq T^{*} \\
\sum_{j=1}^{k_{i}} x_{i j}=q_{i}, \quad 1 \leq i \leq n, \\
x_{i j} \geq 0, \quad 1 \leq i \leq n, \quad 1 \leq j \leq k_{i}, \quad x_{i j} \in\{0\} \bigcup N .
\end{array}\right.
$$

A third option of optimization problem could be convolution of criteria: 


$$
\left\{\begin{array}{l}
\alpha C\left(x_{11}, x_{12}, \ldots, x_{1 k_{1}}, \ldots, x_{n 1}, x_{n 2}, \ldots, x_{n k_{n}}\right)+ \\
\quad(1-\alpha) T\left(x_{11}, x_{12}, \ldots, x_{1 k_{1}}, \ldots, x_{n 1}, x_{n 2}, \ldots, x_{n k_{n}}\right) \rightarrow \min \\
C\left(x_{11}, x_{12}, \ldots, x_{1 k_{1}}, \ldots, x_{n 1}, x_{n 2}, \ldots, x_{n k_{n}}\right) \leq C^{*} \\
T\left(x_{11}, x_{12}, \ldots, x_{1 k_{1}}, \ldots, x_{n 1}, x_{n 2}, \ldots, x_{n k_{n}}\right) \leq T^{*} \\
\sum_{j=1}^{k_{i}} x_{i j}=q_{i}, \quad 1 \leq i \leq n, \\
x_{i j} \geq 0, \quad 1 \leq i \leq n, \quad 1 \leq j \leq k_{i}, \quad x_{i j} \in\{0\} \cup N
\end{array}\right.
$$

Here $a \in[0 ; 1]$ represents significance of the respective criterion.

All involved optimization models are considered as problems of linear integer programming, which requires application of proper mathematical techniques and tools for analysis.

When solving problems (7), (8) and (9) we define both the optimum number of program calls for meeting production target $\left(q_{1}, q_{2}, \ldots, q_{n}\right)$ and the required tool assemblies. In this regard we could consider two options of control over a number of assemblies.

Assume $x=\left(x_{11}, x_{12}, \ldots, x_{1 k_{1}}, \ldots, x_{n 1}, x_{n 2}, \ldots, x_{n k_{n}}\right)$ is an optimum plan calculated through one of the considered models (7), (8) or (9). This way, a demand for tool assembly is $b_{i j s}=a_{i j s} x_{i j}, 1 \leq i \leq n, 1 \leq j \leq k_{i}, 1 \leq s \leq m_{i j}$. Assume a shop has $b^{*}{ }_{i j s}$ assemblies available, $1 \leq i \leq n, 1 \leq j \leq k_{i}, 1 \leq s \leq m_{i j}$. Then an advanced plan to provide tool assemblies is defined by $d_{i j s}=\max \left\{0, b_{i j s}-b_{i j s}^{*}\right\}, 1 \leq i \leq n, 1 \leq j \leq k_{i}, 1 \leq s \leq m_{i j}$.

In some cases, a prompt advanced tool assembly is unrealizable at an enterprise. Then a problem should be solved with regard to a limited number of assemblies:

$$
a_{i j s} x_{i j} \leq b_{i j s}^{*}, 1 \leq i \leq n, 1 \leq j \leq k_{i}, 1 \leq s \leq m_{i j}
$$

If we add condition (10) to one of the tasks (7), (8) or (9) we could interpret this tasks as a search for an optimum number of calls of $\mathrm{NC}$ programs regarding available tool assemblies.

Solution of the stated problems requires input data, namely, lists of tools used for manufacturing of certain parts, monthly production target for parts manufacturing, immediate availability of tools, list of tools to be used with certain machines, etc.

Enterprises often use the same tool assemblies simultaneously for different machines. Warehouse stock of the enterprise may not hold the required quantity of replacement tools, and their order and purchase could take a long time. We need to define the most frequently used tools to adjust purchase requests and a sequence of manufacturing of parts.

Enterprises often use automated systems to create 3D models of tool assemblies and store information about them in enterprise databases. However, these programs can neither analyze the demand for tools to meet the production target, nor track immediate availability of tools.

To identify tools that are used the most based on parts production target, a software program was developed. Production target for a month, a list of tools for CAM-projects and a list of tools installed on machines are input at program entry. At program exit, the user receives a list of tools required for each unit of a machine, ranking of frequently used tool 
assemblies in CAM-projects and information about tools that need to be installed on the machine or uninstalled from it.

This software program provides input data for problems (7), (8) and (9).

Application of the developed software program provided for significant reduction of machine down time associated with replacement and preparation of tool assembly (5-8\% of total machining time). It also contributed to adjustment of a procurement plan for cutting tools promoting increase in orders of frequently used tools, thus avoiding NC program updates due to the lack of required tools in stock (5\% of labor costs for updates of $\mathrm{NC}$ programs).

The proposed model of control over cutting tool demand for machining shops optimizes warehouse stock of multiproduct manufacture according to principles of "just-in-time" and "at specified cost price" through solution of optimization problems (7), (8), (9). Application of this model is instrumental in enhancing efficiency of planning of cutting tool procurement and reducing the acquisition costs. A mechanism of linear integer programming is proposed for application in the software program implementing this model.

This work is completed by state order from the Ministry of Education and Science of the Russian Federation (No.2.1816.2017/ PCh).

\section{References}

1. O.G. Turovets, Management of Manufacturing Processes and Enterprise Administration, 506 (INFRA-M, 2011) ISBN: 978-5-16-004331-9

2. G.N Kanevsky, A.S Shvarev, Works of NSTU n.a. R.E. Alekseyev, Prediction method of automated flow cutting tools for CNC machine tools, No. 2(117), pp. 133-141 (2017) ISSN: 1816-210X 